\title{
FREE GROUPS, HIRSCH-PLOTKIN RADICALS, AND APPLICATIONS TO GEOMETRY
}

\author{
L. AUSLANDER AND E. SCHENKMAN ${ }^{1}$
}

M. Auslander and R. Lyndon (see [5] for a reference) studied properties of $F /[R, R]$, where $F$ is a free group satisfying

$$
1 \rightarrow R \rightarrow F \rightarrow f \rightarrow 1 \text {. }
$$

Their techniques were homological in nature. Shortly thereafter $\mathrm{L}$. Auslander and M. Kuranishi [1] showed that the above-mentioned results have a geometric interpretation. Recently both $\mathrm{H}$. Neumann (see [5] for a reference) and B. H. Neumann [5] returned to the study of the paper of Auslander and Lyndon and gave both nonhomological proofs and stronger results. The first part of the present paper gives a simple proof of a slightly stronger result (Theorem 2 below) than that given by B. H. Neumann (cf. Theorem 2.7 of [5]). The second part extends the geometric interpretation of $\mathrm{L}$. Auslander and M. Kuranishi to this stronger result.

1. The group theory. Let $R$ be a normal subgroup of a free group $F$, let $R^{\prime}$ denote $[R, R]$, and let bars denote the images of $F$ onto $F / R^{\prime}$. We first prove the following two facts.

THEOREM 1. $\bar{F}$ is torsion-free.

TheOREM 2. $\bar{R}$ is the Hirsch-Plotkin radical of $\bar{F}$.

By the Hirsch-Plotkin radical of a group, we mean its maximal locally nilpotent normal subgroup.

If $R^{2}$ also denotes $R^{\prime}$ and if $R^{k}$ is defined inductively to be $\left[R^{k-1}, R\right]$, then we have the following corollary.

Corollary 1. For each integer $k>1, F / R^{k}$ is torsion-free, and $R / R^{k}$ is the Hirsch-Plotkin radical of $F / R^{k}$.

Proof of Corollary 1. Since $R / R^{k}$ is torsion-free, it follows that if $F / R^{k}$ is not torsion-free, there is an $f \in F, f \notin R$, so that for some integer $n, f^{n} \in R^{k}$. But then $\bar{f}^{n}=1$ contrary to Theorem 1 . It is clear that $R / R^{k}$ is in the Hirsch-Plotkin radical $H / R^{k}$ of $F / R^{k}$. If $H / R^{k}$ properly contained $R / R^{k}$ then $H / R^{2}$ would properly contain $R / R^{2}$ contrary to Theorem 2 .

Received by the editors June 11, 1963.

1 The authors are indebted to the National Science Foundation for support and to the referee for his comments. 
Proof of Theorem 1. Suppose that $x^{n} \in R^{\prime}$ for some $x \in F$ and some positive integer $n$. Let $S=\operatorname{Sgp}(R, x)$. Then $R$ is normal in $S$ and $S / R$ is cyclic; consequently $R \geqq S^{\prime}$. On the other hand $S \geqq R$ and hence $S^{\prime} \geqq R^{\prime}$. Since $x^{n} \in R^{\prime}, x^{n} \in S^{\prime}$. But $S$ is a free group and hence $x \in S^{\prime}$ (since $S / S^{\prime}$ is torsion-free). Thus $x \in R$ since $R \geqq S^{\prime}$. But $x \in R$ and $x^{n} \in R^{\prime}$ imply that $x \in R^{\prime}$ (since $R / R^{\prime}$ is torsion-free). Hence $x \in R^{\prime}$ and $\bar{F}$ is torsion-free as is asserted in Theorem 1 .

Before giving the proof of Theorem 2 we define the Schreier property and state a well-known fact as a lemma (cf. [2] for a proof for instance).

The transversal $T$ of the subgroup $H$ of the free group $F$ on a free set of generators $X$, has the Schreier property if whenever $y_{1} \cdots y_{n}$ is a reduced word in $T$ (with each $y_{i}$ an element of $X$ or its inverse), then $y_{1} \cdots y_{n-1}$ is also in $T$.

Lemma. Let $X$ be a free set of generators of a free group $F$, let $H$ be a nontrivial subgroup of $F$, and let $T$ be a transversal of $H$ in $F$ with the Schreier property. Then the set of nontrivial elements of $T X T^{-1} \cap H$ is a free set of generators of $H$.

Proof of Theorem 2. Let $\bar{H}$ be the Hirsch-Plotkin radical of $\bar{F}$. If $\bar{x} \in \bar{H}$ and $\bar{b} \in \bar{R}$ then for some integer $m$ (depending on $\bar{x}$ and $\bar{b}$ ), $[\cdots[b, \bar{x}], \cdots, \bar{x}]=1$, the bracket taken $m$ times, or equivalently, if $h$ and $x$ are counter images in $F$ then $[\cdots[b, x], \cdots, x] \in R^{\prime}$, the bracket taken $m$ times. We shall derive a contradiction to this last relation on the assumption that $\bar{H}$ properly contains $\bar{R}$, or equivalently that the counter image $H$ of $\bar{H}$ properly contains $R$. Suppose then that $y \in H, y \notin R$. Let $x \notin R$ be a member of a free set of generators of $\operatorname{Sgp}(y, R)$ and let $S=\operatorname{Sgp}(x, R)$. Then $S$ is a free subgroup of the free group $\operatorname{Sgp}(y, R)$ and hence by the lemma (since 1 is in the transversal) $x$ is a member of a free set of generators of $S$. But then each of the other free generators of this set may be modified by a suitable power of $x$ to produce a set of free generators of $S$ consisting of $x$ and a set $B$ of elements $b_{\alpha}$ which are in $R$.

Now $S / R$ is cyclic and either of finite order $n$ or of infinite order; in the first case $T_{n}=\left\{1, x^{-1}, \cdots, x^{-n+1}\right\}$ and in the second case $T_{\infty}=\left\{1, x, x^{-1}, x^{2}, x^{-2}, \cdots\right\}$ is a transversal of $R$ in $S$ with the Schreier property. We use the lemma again to pick a free set of generators of the subgroup $R$ of the free group $S$. When $T_{n}$ is the transversal, this set will consist of $x^{n}$ and the conjugates $b_{\alpha}^{x^{i}}$ for $b_{\alpha} \in B$ and $i=0,1, \cdots, n-1$. When $T_{\infty}$ is the transversal, it will consist of $b_{\alpha}^{x^{i}}$ for $b_{\alpha} \in B$ and all integers $i$.

We will now have our contradiction when we show that if $b$ is one 
of the $b_{\alpha}$ then $[\cdots[b, x], \cdots, x]$, the bracket taken $m$ times, is not in $R^{\prime}$ for each natural number $m$. Indeed $[b, x]=b^{-1} b^{x} ;[[b, x], x]$ $=b^{-x} b\left(b^{-1} b^{x}\right)^{x}$ which is congruent to $b^{x^{2}}\left(b^{-x}\right)^{2} b \bmod R^{\prime}$. A direct induction then gives that $[\cdots[b, x], \cdots, x]$, the bracket taken $m$ times, is congruent to

$$
\prod_{i=0}^{m}\left(b^{x^{m-i}}\right)^{(-1)^{i}\left(\begin{array}{l}
m \\
i
\end{array}\right)} \bmod R^{\prime} .
$$

When $T_{\infty}$ is the transversal (and also when $T_{n}$ is the transversal with $m=1$ ), the elements $b^{x^{m-i}}$ are free generators of $R$, and hence the product in the last expression is obviously not in $R^{\prime}$. In case $T_{n}$ is the transversal with $m>1$, we let $\epsilon$ denote a primitive $n$th root of 1 and note that $(1-\epsilon)^{m} \neq 0$. We expand this expression by the binomial theorem and combine like powers of $\epsilon$ after using only the relation $\epsilon^{n}=1$ wherever possible. Since $(1-\epsilon)^{m} \neq 0$, it follows that it is not possible for all the coefficients of the $\epsilon_{i}$ to be zero after the combinations described. Looking back at the product describing

$$
[\cdots[b, x], \cdots, x] \bmod R^{\prime}
$$

the bracket taken $m$ times, we see that this product cannot be in $R^{\prime}$ as was to be shown. This proves the theorem.

2. The applications to geometry. Let $\Gamma$ be a torsion-free finitely generated group with a nilpotent Hirsch-Plotkin radical $N$ so that $f=\Gamma / N$ is finite. We will call such a group $\Gamma$ a nil-admissible group; thus a nil-admissible group $\Gamma$ satisfies the diagram

$$
1 \rightarrow N \rightarrow \Gamma \rightarrow f \rightarrow 1
$$

with $f$ a finite group. By a theorem of $\mathrm{Mal}^{\prime} \mathrm{cev}$ [3] there is a unique connected, simply connected, nilpotent Lie group $L(N)$ with the following properties:

(1) $N \leqq L(N)$.

(2) $L(N) / N$ is compact.

(3) Every automorphism of $N$ is uniquely extendable to $L(N)$. Now it is straightforward to verify that if we have an exact sequence of groups

$$
1 \rightarrow A \rightarrow B \rightarrow C \rightarrow 1
$$

and $A$ is contained in a group $A^{*}$ with the property that every automorphism of $A$ is uniquely extendable to an automorphism of $A^{*}$, then there exists a unique $B^{*}$ satisfying the diagram 


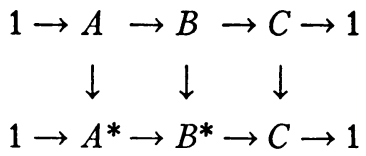

where all vertical arrows are inclusions. Hence we have a group $\Gamma^{*}$ satisfying the diagram

$$
1 \rightarrow L(N) \rightarrow \Gamma^{*} \rightarrow f \rightarrow 1
$$

where the vertical arrows are inclusions. But $\Gamma^{*}$ is now a split extension; i.e., $\Gamma^{*}=L(N) \cdot f$. This follows directly from the divisibility of $L(N)$ or from a result of Mostow [4]. Hence $\Gamma^{*} \leqq L(N) \cdot A(L(N))$, where $A(L(N))$ denotes the group of continuous automorphisms of $L(N)$. But we can define $L(N) \cdot A(L(N))$ as a group of transformations of $L(N)$ by first defining the map $\psi$ so that $\psi(n, g)=n$ and then defining $(n, g) m=\psi((n, g)(m, e))$ where $n, m \in L(N), g \in A(L(N))$ and $e$ is the identity element of $A(L(N))$. We will call this the affine group of the nilpotent Lie group $L(N)$. Thus $\Gamma^{*}$ has been interpreted as a subgroup of affinities of $L(N)$. Since:

(1) $\Gamma / N$ is finite,

(2) $L(N) / N$ is a compact manifold,

(3) $\Gamma$ is torsion-free,

it is straightforward to verify that $\Gamma$ operates properly discontinuously on $L(N)$ and with compact fundamental domain. We have therefore proven the following theorem.

TheOREM 3. If $\Gamma$ is nil-admissible then $\Gamma$ operates properly discontinuously and with compact fundamental domain on a topological euclidean space.

CoROllaRY 2. Let $G$ be any module on which $\Gamma$ operates trivially. Then the cohomology groups vanish, $H^{q}(\Gamma, G)=0$ for all $q>\operatorname{dim} L(N)$.

In view of Corollary 1 , when a normal subgroup $R$ has finite index in a finitely generated free group $F$, then $F / R^{k}$ is nil-admissible and hence Theorem 3 is directly applicable to $\Gamma=F / R^{k}$.

Added in proof. It has recently come to our attention that some of our results are closely related to results already in the literature. Thus Theorem 1 is due to G. Higman and is also a very special case of Theorem 5 in Baumslag, Wreath products and extensions, Math. Z. 81 (1963), 286-289. Theorem 2 is very closely related to Lemma 3 of Gruenberg, The residual nilpotence of certain presentations of finite groups, Arch. Math. 13 (1962), 408-417. 


\section{BIBLIOGRAPHY}

1. L. Auslander and M. Kuranishi, On the holonomy group of locally Euclidean spaces, Ann. of Math. (2) 65 (1957), 411-415.

2. M. Hall, Theory of groups, Macmillan, New York, 1959.

3. A. Mal'cev, On a class of homogeneous spaces, Amer. Math. Soc. Transl. (1)9 (1951), 276-307.

4. G. D. Mostow, Self-adjoint groups, Ann. of Math. (2) 62 (1955), 44-45.

5. B. H. Neumann, On a theorem of Auslander and Lyndon, Arch. Math. 13 (1962), 4-9.

Purdue University

\section{IN JECTIVE MODULES UNDER CHANGE OF RINGS ${ }^{1}$}

\section{ERNST SNAPPER}

Introduction. Let $U$ and $V$ be rings with unit element and $k: U \rightarrow V$ a ring epimorphism with kernel $I$. Every $V$-module (all modules are left modules) can be regarded as a $U$-module under $k$ and hence it makes sense to ask when a $V$-module is $U$-injective.

For $u \in U$, we denote the left ideal $\{c \mid c \in U, c u=0\}$ by $0: u$. The answer to the above question is simply:

Criterion. $A V$-module $A$ is $U$-injective if and only if it satisfies the following two conditions:

(1) $A$ is V-injective;

(2) If $u \in I$ and $a \in A$ and $(0: u) a=0$, then $a=0$. (The cutest way to put (2) is $0:(0: u)=0$ for all $u \in I$.)

We prove the criterion in $\S 1$ and make an application of it to $G$ modules in $\$ 2$. ( $G$ stands for a finite group.)

1. Proof of the criterion. Let the $V$-module $A$ be $U$-injective. In order to prove condition 1 , we select a left ideal $M$ of $V$ and a $V$ homomorphism $g: M \rightarrow A$. We have to produce an element $a \in A$ such that $g(v)=v a$ for all $v \in M$. (See [1, Theorem 3.2, p. 8].) Hereto we consider the left ideal $k^{-1}(M)$ of $U$ and the $U$-homomorphism $g k: k^{-1}(M) \rightarrow A$. Since $A$ is $U$-injective, there exists an $a \in A$ such that $g k(u)=u a$ for all $u \in k^{-1}(M)$. Let now $v \in M$. Since $k$ is an epi, $v=k(u)$ for some $u \in k^{-1}(M)$ and, hence, $g(v)=g k(u)=u a$. The action of $U$ on $A$ is such that $u a=k(u) a=v a$ and condition 1 is proved.

Received by the editors March 18, 1964.

1 This research was supported in part by the National Science Foundation grant NSF-GP722. 